\title{
Periacetabular Brucella Osteomyelitis
}

\author{
Hakan Cift ${ }^{1}$, Krishna Reddy ${ }^{2}$, Esat Uygur ${ }^{3}$, Salih Söylemez ${ }^{3}$, Serkan Şenol ${ }^{4}$, Korhan Ozkan ${ }^{3}$, \\ Maria Silvia Spinelli ${ }^{5}$, Abdullah Eren ${ }^{6}$
}

${ }^{1}$ Orthopaedics and Traumatology Department, Medipol University, Istanbul, Turkey; ${ }^{2}$ Orthopaedics and Traumatology Department, Royal Orthopaedic Hospital, Birmingham, UK; ${ }^{3}$ Orthopaedics and Traumatology Department, Medeniyet University Goztepe Research and Training Hospital, Istanbul, Turkey; ${ }^{4}$ Department of Pathology, Medeniyet University Goztepe Research and Training Hospital, Istanbul, Turkey; ${ }^{5}$ Orthopaedics and Traumatology Department, University Hospital Agostino Gemelli, Catholic University of the Sacred Heart School of Medicine, Rome, Italy; ${ }^{6}$ Orthopaedics and Traumatology Department, Florence Nightingale Hospital, Istanbul, Turkey.

Email: hakanturancift@yahoo.com

Received December $23^{\text {rd }}, 2012$; revised February $5^{\text {th }}, 2013$; accepted February $26^{\text {th }}, 2013$

\begin{abstract}
Introduction: Although Brucellosis has a limited geographic distribution; it remains a challenge in certain parts of the world such as in Mediterranean, western Asian, Latin American and African regions. We present a unique case of periacetabular Brucella osteomyelitis and increase awareness of possible widespread distrubition of Brucella osteomyelitis and its ability to affect any region of the musculoskeletal system. Case Presentation: A 44-year-old male farmer presented with symptoms of pain radiating from his left hip to his thigh of five years duration. There was a history of night sweats and fever for the past two months. A lytic area with smooth borders in left periacetabular region was detected on pelvic roentgenography of the patient. Magnetic resonance imaging revealed a cavitatory lesion in relation to hip joint. Open biopsy was undertaken with the differential diagnosis of an infectious (Brucella or Tuberculous) or tumoral lesion. Intraoperative frozen sections showed granulomatous inflammatory tissue. Post debridement, the cavity was filled with autograft taken from the patient's right iliac wing. Postoperative immunohistochemistry confirmed diagnosis of Brucella osteomyelitis. Oral Doxycyline, Rifampicine and Ciprofloxacin were administered for 3 months. At one-year postoperatively, the patient had a painless, unrestricted range of motion and function in relation to the affected hip. Conclusion: In endemic regions, Brucella osteomyelitis should be considered in differential diagnosis in patients with arthralgia and/or spondylodiscitis in the presence of radiologically suspected osseous lesions.
\end{abstract}

Keywords: Brucella; Acetabulum; Osteomyelitis

\section{Introduction}

Brucella is a Gram-negative coccobacillus [1]. Although the clinical presentation of Brucella is usually non-specific, its musculoskeletal involvement is frequent [1-2]. The frequency of osteoarticular involvement of Brucella ranges between $10 \%-85 \%$ and the most frequent osteoarticular involvements are seen in the form of arthritis, spondylitis, bursitis, tenosynovitis and osteomyelitis [3-6]. Brucella osteomyelitis may appear as a radiolucent area and it may be confused with tumour lesion on radiographs. Thus, care should be given during diagnosis of etiology of the lesions [7].

\section{Case Presentation}

A 44-year-old male farmer and animal breeder presented to our hospital with complaints of severe hip pain limiting his ability to walk, five years history of left hip/thigh pain radiating to his calf. The patient was initially exam- ined in different centers and he had been diagnosed with lumbar disc disease and treated as such with anti-inflammatory medication with no relief. $\mathrm{He}$ also had two-month history of night pain and fever. Physical examination of the patient revealed severe pain during internal and external rotation of his left hip. A well-circumscribed lytic lesion was seen in the patient's left peri-acetabular region on radiograph (Figure 1). Magnetic Resonance (MR) imaging on T2 sequences demonstrated a caviatory fluid filled lesion in the anterosuperior acetabular ridge in the left peri-acetabular region (Figure 2).

The patient was hospitalized with a possible diagnosis of infectious (Tuberculosis/Brucella osteomyelitis) or a neoplastic lesion.

Further examination of the patient displayed a C-reactive protein of $6.35 \mathrm{mg} / \mathrm{dL}$ (Normal: $0.00-0.800$ ). The patient underwent an open biopsy. The lytic area was accessed by an incision from the superolateral acetabu- 
lum using anterior (Smith-Peterson) approach. Intraoperative frozen sections revealed granulomatous inflamematory focus with sequestrum. Debridement was performed and the cavity packed with autograft from the right iliac crest. The material was then sent for a routine pathological analysis and the result was chronic inflamematory fibro-lipomatous tissues (Figure 3). Subsequent immunonological tests confirmed a diagnosis of Brucella osteomyelitis- Brucella agglutination test (Rose-Bengal), Brucella IgG \& IgM titers 2.599 (index < 0.593) \& 0.773 (index $<0.563$ ) respectively] and Brucella Agglutination (Coombs Antiserum) $\geq 1 / 160$ were all positive. The patient was commenced on a triple oral combination therapy of doxycycline, rifampicin and ciprofloxacin for three months. Joint movement exercises were initiated after the surgery and weight bearing was restricted for 6 weeks. The patient has made a full recovery of range of joint movement and function postoperatively with resolution of symptoms at his last follow-up visit one year postoperatively. In addition, his CRP and Brucella Agglutination (Coombs Antiserum) values have reverted back to normal.

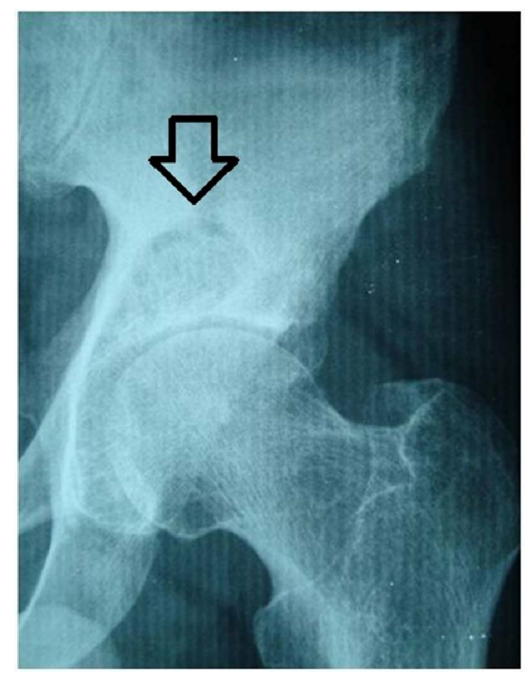

Figure 1. Osteolytic lesion in the superomedial acetabulum.

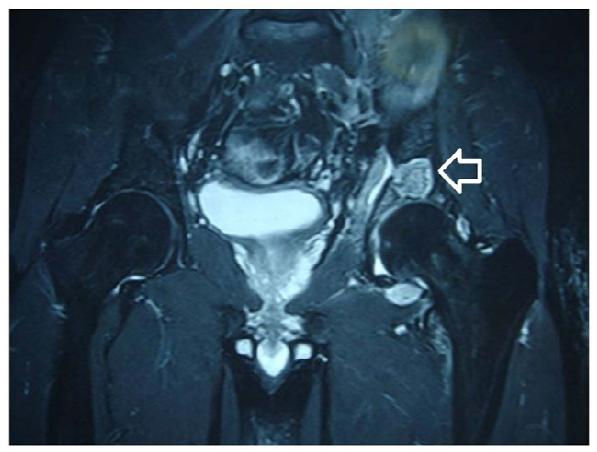

Figure 2. Osteolytic lesion in coronal T2-weighted sequence MR image.

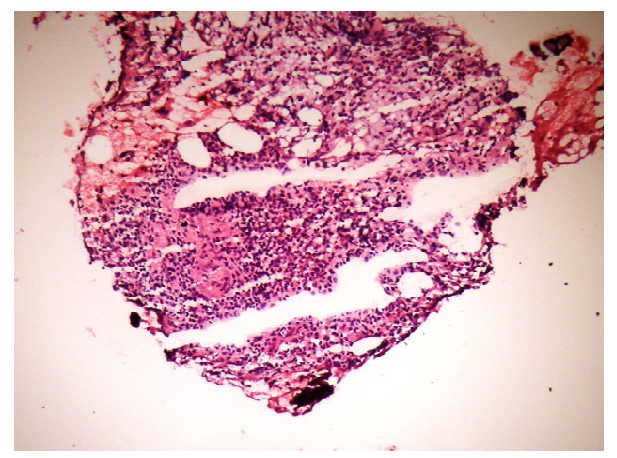

Figure 3. Microscopic image of the sample amplified $\times 100$ after hemotoxylin and eosine staining.

\section{Discussion}

Brucellosis is a disease frequently seen in domestic and wild animals. Brucella infections may often be seen in humans, but they do not play a role in the natural progression of the disease or in the life cycle of the microorganisms [2]. Brucella infections are endemically seen in Middle and East Mediterranean region including Turkey, Arabian Peninsula, Middle and Southeast Asia and Middle and South America.

Brucellosis is particularly a reproductive system disease in animals. Four of the six types that cause the disease in animals (B. abortus, B. melitensis, B. suis and more rarely $B$. canis) are pathogenic in humans. Intake of 10 to 100 bacteria through inhalation is sufficient to make the disease [2]. Development of the disease in normal circumstances is often through use of unpasteurized milk or dairy products. Mortality due to brucellosis is low $(5 \%)$ in humans, but it may cause severe clinical conditions such as meningitis and endocarditis [8]. The disease may have non-specific symptoms such as complaints associated with the musculoskeletal system, fatigue, sweating, prolonged fever periods, anxiety or depression, and hepato-biliary or gastrointestinal complaints.

It was reported in the study made by Pourbagher and associates on 251 patients that the most frequent complaints were due to osteo-articular involvement and spondylitis was the second frequent condition following sacroiliac joint involvement. In another study made by Tasova et al. on 238 patients in Turkey, the rate of musculoskeletal system involvement was found to be $36.5 \%$ $69 \%$ and the most frequently involved joint was again the sacroiliac joint [9-11].

Brucella osteomyelitis may imitate other diseases of the bone and should be considered during differential diagnosis as it epidemiologically occurs in the same endemic regions as tuberculosis. A biopsy is essential to differentiate it from neoplastic diseases that may as well present as lytic lesions and may be impossible to distinguish on radiological appearances. 
Brucella osteomyelitis is more likely to affect the axial skeleton. However, a multifocal Brucella osteomyelitis involved in both tibia and humerus has also been reported [12]. The peri-acetabular involvement as in our case has not been reported in English literature. Brucellosis may present with a lytic lesion mimicking a tumour.

Laboratory findings of Brucella infection are generally variable. A normal white cell count is observed in $80 \%$ of patients [13]. Erythrocyte sedimentation shows an increase only in $30 \%$ of patients [13]. It is quite difficult to isolate Brucella in the specimens taken. A serum agglutination test is routinely used for an initial screening of Brucella infection and a Coomb's test supports the results of the serum agglutination test [13].

\section{Conclusion}

We believe this case is probably the first report of osteomyelitis of the acetabular region secondary to Brucellosis in English literature. Many of the patients with Brucella osteomyelitis may have haematological parameters within normal limits.

\section{Clinical Message}

Brucella osteomyelitis must definitely be considered in differential diagnosis if a suspicious lesion is noted on radiographic examination in patients with arthralgia and/or spondylodiscitis in endemic regions; especially in individuals who consume unpasteurized milk and in those whose occupation involves animal breeding.

\section{REFERENCES}

[1] G. L. Mandell, "Principles and Practice of Infectious Diseases," 5th Edition, Philadephia, 2000, pp. 2386-2393.

[2] W. J. Koopman, "Arthritis and Allied Conditions," 14th Edition, Philadelphia, 2001, p. 2576.

[3] A. R. Mousa, S. A. Muhtaseb, D. S. Almudallal, S. M. Khodeir and A. A. Marafie, "Osteoarticular Complications of Brucellosis: A Study of 169 Cases," Reviews of Infectious Diseases, Vol. 9, No. 3, 1987, pp. 531-543. doi:10.1093/clinids/9.3.531
[4] Y. Weil, Y. Mattan, M. Liebergall and G. Rahav, "Brucella Prosthetic Joint Infection: A Report of 3 Cases and a Review of the Literature," Clinical Infectious Diseases, Vol. 36, No. 7, 2003, pp. 81-86. doi:10.1086/368084

[5] S. G. Gundes, H. Gundes, A. Sarlak and A. Willke, "Primary Brucellar Psoas Abscess: Presentation of a Rare Case of Psoas Abscess Caused by Brucella melitensis without any Osteoarticular Involvement," International Journal of Clinical Practice, Vol. 147, 2005, pp. 67-68.

[6] L. Cecchini, G. Coari, A. Iagnocco and G. Valesini, "Brucellar Spinal Abscess: Case Report," Reumatismo, Vol. 53, No. 3, 2001, pp. 229-231.

[7] T. P. Fowler, J. Keener and J. A. Buckwalter, "Brucella Osteomyelitis of the Proximal Tibia" The Iowa Orthopaedic Journal, Vol. 24, 2004, pp. 30-32.

[8] N. Sayar, S. Terzi, H. Y. Yilmaz, H. Atmaca, F. Kocak, S. U. Dayi, N. Cakmak, A. Tarhan, A. Ozler and K. Yesilcimen, "A Case of Prosthetic Mitral Valve Brucella Endocarditis Complicated with Torsades de Pointes," Heart and Vessels, Vol. 21, No. 5, 2006, pp. 331-333. doi:10.1007/s00380-006-0907-3

[9] H. Turan, K. Serefhanoglu and E. Karadeli, "A Case of Brucellosis with Abscess of the Iliacus Muscle, Olecranon Bursitis, and Sacroilitis," International Journal of Infectious Diseases, Vol. 13, No. 6, 2009, pp. 485-487. doi:10.1016/j.ijid.2009.02.002

[10] Y. Taşova, N. Saltoglu, G. Sahin and H. Z. Aksu, "Osteoarticular Involvement of Brucellosis in Turkey," Clinical Rheumatology, Vol. 18, No. 3, 1999, pp. 214-219. doi:10.1007/s100670050087

[11] A. Pourbagher, M. A. Pourbagher, L. Savas, T. Turunc, Y. Z. Demiroglu, I. Erol and D. Yalcintas, "Epidemiologic, Clinical, and Imaging Findings in Brucellosis Patients with Osteoarticular Involvement," American Journal of Roentgenology, Vol. 187, No. 4, 2006, pp. 873-880. doi:10.2214/AJR.05.1088

[12] A. Zwass, F. Feldman. "Case report 875: Multifocal Osteomyelitis-A Manifestation of Chronic Brucellosis," Skeletal Radiology, Vol. 23, No. 8, 1994, pp. 660-663. doi:10.1007/BF02580393

[13] G. F. Araj, "Update on Laboratory Diagnosis of Human Brucelloscis," International Journal of Antimicrobial Agents, Vol. 36, Suppl. 1, 2010, pp. 12-17. doi:10.1016/j.ijantimicag.2010.06.014 